\title{
Sago Starch-Mixed Low-Density Polyethylene Biodegradable Polymer: Synthesis and Characterization
}

\author{
Md Enamul Hoque, ${ }^{1}$ Tan Jie Ye, ${ }^{1}$ Leng Chuan Yong, ${ }^{1}$ and KhairulZaman Mohd Dahlan ${ }^{2}$ \\ ${ }^{1}$ University of Nottingham Malaysia Campus, 43500 Semenyih, Selangor, Malaysia \\ ${ }^{2}$ Malaysian Nuclear Agency, 43000 Kajang, Selangor, Malaysia \\ Correspondence should be addressed to Md Enamul Hoque; enamul.hoque@nottingham.edu.my
}

Received 17 January 2013; Revised 11 June 2013; Accepted 12 June 2013

Academic Editor: Guoping Chen

Copyright (c) 2013 Md Enamul Hoque et al. This is an open access article distributed under the Creative Commons Attribution License, which permits unrestricted use, distribution, and reproduction in any medium, provided the original work is properly cited.

\begin{abstract}
This research focuses on synthesis and characterization of sago starch-mixed LDPE biodegradable polymer. Firstly, the effect of variation of starch content on mechanical property (elongation at break and Young's modulus) and biodegradability of the polymer was studied. The LDPE was combined with $10 \%, 30 \%, 50 \%$, and $70 \%$ of sago for this study. Then how the cross-linking with trimethylolpropane triacrylate (TMPTA) and electron beam (EB) irradiation influence the mechanical and thermal properties of the polymer was investigated. In the 2nd study, to avoid overwhelming of data LDPE polymer was incorporated with only $50 \%$ of starch. The starch content had direct influence on mechanical property and biodegradability of the polymer. The elongation at break decreased with increase of starch content, while Young's modulus and mass loss (i.e., degradation) were found to increase with increase of starch content. Increase of cross-linker (TMPTA) and EB doses also resulted in increased Young's modulus of the polymer. However, both cross-linking and EB irradiation processes rendered lowering of polymer's melting temperature. In conclusion, starch content and modification processes play significant roles in controlling mechanical, thermal, and degradation properties of the starch-mixed LDPE synthetic polymer, thus providing the opportunity to modulate the polymer properties for tailored applications.
\end{abstract}

\section{Introduction}

The development of innovative biopolymers has been underway for a number of years and continues to be an area of interest for the modern scientists. In 1996, shipments from the Canadian Plastic Industry increased by $10.6 \%$ from 1995 levels to $\$ 9.1$ billion $[1,2]$. As reported by Fomin [3], worldwide production of synthetic polymers reached 130 million tons per year at the end of the 20th century, while the demand for biodegradable polymer is reported to be growing by $30 \%$ each year $[4,5]$. Meanwhile, Europe has an estimated average usage of $100 \mathrm{~kg}$ of polymer per person each year [6]. Polyethylene (PE) is widely used in medical devices and pharmaceutical packaging [7]. Different types of polymers such as high-density PE (HDPE), low-density PE (LDPE), or linear low-density PE (LLDPE) offer different benefits and functionalities. HDPE provides stiffness, chemical resistance, and barrier properties, while LDPE offers resistance to stress cracking and excellent impact properties [8]. In addition, its capability of being sterilized is a favourable and an important aspect. Cross-linking is a broadly used method for modifying the polymer properties. This process involves the formation of tridimensional structures that cause substantial changes [9]. The mechanical property of biomaterial is equally important as biocompatibility since an inadequate performance or premature failure of the implants might impose serious health issues to the patients [10-15]. Besides, most of the body implants undergo dynamic loading that might affect the mechanical behaviour (e.g., creep or relaxation) of the implants. The implants should maintain their stability in the body for a prescribed time as required [16]. Therefore, the durability or long-term mechanical behaviour of new biomaterials has become a prime concern for their adoption to medical devices.

Starch is a hydrocolloid biopolymer that can be found in a variety of agricultural feedstocks such as wheat, corn, rice, beans, and potatoes $[17,18]$. Starch consists of one branched and linear polymer [19] and is usually found in the 
TABLE 1: Relative percentage of LDPE and starch of polymer samples.

\begin{tabular}{lcc}
\hline Sample ID & \% LDPE & \% Starch \\
\hline LDPE00-Starch0 & 100 & 0 \\
LDPE90-Starch10 & 90 & 10 \\
LDPE70-Starch30 & 70 & 30 \\
LDPE50-Starch50 & 50 & 50 \\
LDPE30-Starch70 & 30 & 70 \\
\hline
\end{tabular}

form of granules. Different starches and percentages could be employed to modulate polymer properties. A number of studies have reported the development of biodegradable polymer by incorporating starch into LDPE and the characterization of these synthesized polymers [20-27]. These studies mostly focused on investigating the influences of starch content and so forth on the mechanical and degradation properties of the polymer. However, too little attention had been paid so far to evaluate the effects of modification processes (e.g., addition of cross-linker and electron beam irradiation) on the mechanical and thermal properties of the polymer. In this study, the effects of cross-linking with trimethylolpropane triacrylate (TMPTA) and electron beam (EB) irradiation on mechanical and thermal properties of the polymer were investigated besides the effect of starch content on the mechanical and degradation characteristics of the sago starch-incorporated LDPE polymer.

\section{Materials and Experimental Methods}

2.1. Synthesis of Polymer. In the 1st phase of the experiment, five types of LDPE polymer samples were produced in combination with various percentages of starch. Low-density polyethylene (LDPE) and sago starch (Igan Sago Industries Sdn. Bhd.) were weighed by a toploader balance (AND, model GF-3000) with various percentages of LDPE and starch as reported in Table 1. The weighed LDPE and starch were premixed and blended in a brabender mixer for six minutes at $135^{\circ} \mathrm{C}$. The blends produced from the brabender were then transferred to a hydraulic machine (Lab Tech) for compression. The compression process involved four steps: preheating, venting, pressing, and cooling. First three steps involved hot pressing, while the last step was cold pressing. The samples were placed in the middle of a square steel frame which was then clipped between two other steel plates. There were two thinner and smoother steel plates between these three layers of thicker plates. Polyethylene terephthalate (PET) sheets were used in between polymer surface and steel plate to prevent sticking the polymer samples to the steel plate. The molding temperature and pressure were $150^{\circ} \mathrm{C}$ and $10 \mathrm{MPa}$, respectively. Hot press and cold press processes were conducted using the same machine but different moulds. Upon hot pressing that was conducted at the upper part of the machine, samples were transferred to the bottom part for cold pressing. Mechanical and biodegradation tests were performed over these 1st phase samples.

In the 2 nd phase, the effects of crosslinking with trimethylolpropane triacrylate (TMPTA) and electron beam (EB) irradiation on the mechanical and thermal properties of the
TABLE 2: Polymer composition with TMPTA cross-linker and EB irradiation doses.

\begin{tabular}{lcccc}
\hline Sample ID & $\begin{array}{c}\% \\
\text { LDPE }\end{array}$ & $\begin{array}{c}\% \\
\text { Starch }\end{array}$ & $\begin{array}{c}\% \\
\text { TMPTA }\end{array}$ & $\begin{array}{c}\text { EB irradiation } \\
(\mathrm{kGy})\end{array}$ \\
\hline LDPE50-STARCH50 & 50 & 50 & 0 & 0 \\
TMPTA1-DOSE0 & 50 & 50 & 1 & 0 \\
TMPTA3-DOSE0 & 50 & 50 & 3 & 0 \\
TMPTA5-DOSE0 & 50 & 50 & 5 & 0 \\
TMPTA1-DOSE10 & 50 & 50 & 1 & 10 \\
TMPTA3-DOSE10 & 50 & 50 & 3 & 10 \\
TMPTA5-DOSE10 & 50 & 50 & 5 & 10 \\
TMPTA1-DOSE30 & 50 & 50 & 1 & 30 \\
TMPTA3-DOSE30 & 50 & 50 & 3 & 30 \\
TMPTA5-DOSE30 & 50 & 50 & 5 & 30 \\
TMPTA1-DOSE50 & 50 & 50 & 1 & 50 \\
TMPTA3-DOSE50 & 50 & 50 & 3 & 50 \\
TMPTA5-DOSE50 & 50 & 50 & 5 & 50 \\
TMPTA1-DOSE70 & 50 & 50 & 1 & 70 \\
TMPTA3-DOSE70 & 50 & 50 & 3 & 70 \\
TMPTA5-DOSE70 & 50 & 50 & 5 & 70 \\
TMPTA1-DOSE100 & 50 & 50 & 1 & 100 \\
TMPTA3-DOSE100 & 50 & 50 & 3 & 100 \\
TMPTA5-DOSE100 & 50 & 50 & 5 & 100 \\
\hline
\end{tabular}

polymer samples were investigated. To avoid overwhelming of data, the polymer samples used in this second phase experiments consisted of 50\% LDPE and 50\% starch. Various percentages of TMPTA were added to the LDPE-starch blends during the blending process and then irradiated accordingly with various doses of EB as presented in Table 2. Upon crosslinking, the polymer samples were pressed under the hydraulic machine and irradiated with $\mathrm{EB}$ at the doses of $10 \mathrm{kGy}, 30 \mathrm{kGy}, 50 \mathrm{kGy}, 70 \mathrm{kGy}$, and $100 \mathrm{kGy}$. The energy and current were set at $1.5 \mathrm{MeV}$ and $30 \mathrm{~mA}$, respectively.

2.2. Sample Preparation: Dumb-Bell-Shaped Cutting. Upon completion of synthesis process, the polymer samples were cut into dumb-bell-shaped by a dumb-bell cutter (model SDL-100; Dumb-Bell Co., Ltd.) according to ASTMD 1882L standard. The dumb-bell-shaped samples had smooth surface especially, in the neck section that avoided stress concentration during mechanical test.

2.3. Mechanical Characterization: Tensile Test. Tensile test was carried out to investigate the influence of starch content and further the effect of modification processes, namely, crosslinking with TMPTA and EB irradiation on the polymer's mechanical property. The dumb-bell-shaped sample thicknesses were measured by a thickness gauge (Mitutoyo, model EMD-57B-11M) and were keyed in the test system. Tensile test was performed by using a uniaxial testing system (Instron 3365) and a $5 \mathrm{kN}$ load cell (Canton, MA, USA) according to ASTMD1882L standard. The cross-head speed was set at $10 \mathrm{~mm} / \mathrm{min}$. For each type of polymer, five samples 
were tested. Young's modulus was calculated as stress divided by strain to evaluate the mechanical property of the polymer samples. A Student's $t$-test was performed to compare mean values from all independent sample groups using a Minitab statistics software version 12.2 (Minitab Inc., State College, PA, USA) at a significance level of 0.05 .

2.4. Degradation Study. The in vitro degradation study was performed on the dumb-bell-shaped LDPE polymer samples containing various percentages of starch $(10 \%, 30 \%, 50 \%$, and $70 \%$ ) by burying the polymer samples at the exterior under the soil at a depth of 2 feet for a period of one month. Each type of polymer had five samples. This study was to primarily investigate the variation of degradation kinetics due to the variation of starch content. The degradation phenomenon was evaluated through mass loss and change in surface morphology. Prior to burial, the polymer samples were characterized by weighing and recording their initial mass using an electronic balance with a resolution of $0.1 \mathrm{mg}$. After one month of burial, the samples were dug out and cleaned to ensure complete removal of soil/mud. Samples were then placed in an area with sufficient ventilation for natural drying. The dried degraded samples were weighed using the same electronic balance as carried out before starting degradation. Subsequently, the percentage of mass loss of respective sample was measured as follows:

$$
\% \text { Mass Loss }=\frac{M_{i}-M_{f}}{M_{i}} \times 100,
$$

where, $M_{i}$ is the initial mass (i.e., mass before degradation) and $M_{f}$ is the final mass (i.e., mass after degradation).

\subsection{Thermal Analysis: Differential Scanning Calorimetry} (DSC). The thermal characteristic of cross-linked and EB irradiated polymer sample was determined by differential scanning calorimeter (TA Instruments DSC 2910, New Castle, DE, USA). An indium standard was used to calibrate the instrument. The sample weight of $5 \mathrm{mg}$ was taken for scanning. All samples were placed in aluminum pans and scanned from $-70^{\circ} \mathrm{C}$ to $200^{\circ} \mathrm{C}$ at a rate of $10^{\circ} \mathrm{C} / \mathrm{min}$, using argon as purge gas. Five samples for each type of polymer were analyzed. The DSC analysis provided the melting point of the polymer.

\section{Results and Discussion}

3.1. Mechanical Characteristics. The mechanical experiment (tensile test) initially investigated the influence of starch content and further the effect of modification processes, namely, cross-linking with TMPTA and EB irradiation on the polymer's mechanical property. The starch content and modification process (crosslinking and EB irradiation) had direct influences on the mechanical characteristics of the starch-incorporated LPDE polymer. The elongation at break of the polymer samples decreased with the increase of starch content, while Young's modulus increased with the increase of starch content as demonstrated in Figures 1 and 2. The results are in agreement with the findings of some other

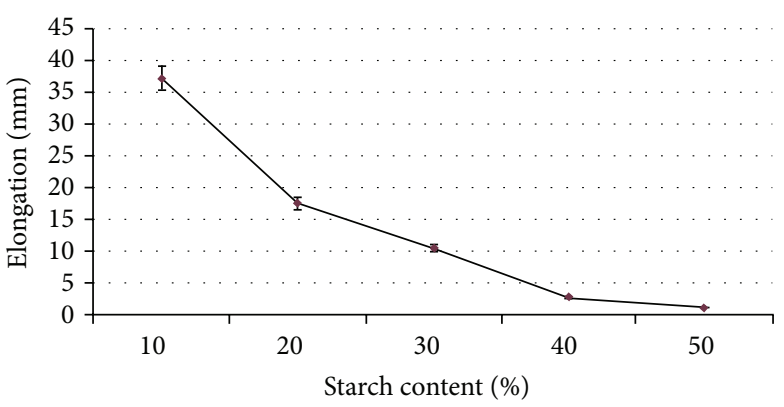

FIGURE 1: Variation of elongation with starch content of LDPE/starch blends without modification.

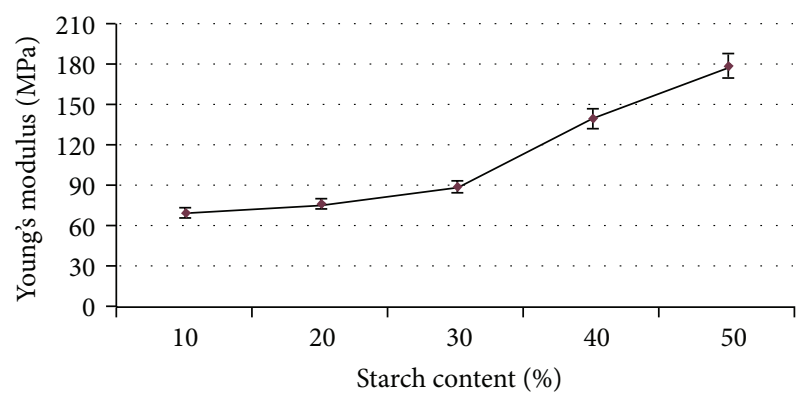

FIgURE 2: Variation of Young's modulus with starch content of LDPE/starch blends without modification.

studies [18-23]. The elongation at break which decreased with the starch content could be attributed to the phenomenon that the starch granule containing hydroxyl groups on its surface is highly hydrophilic, whereas LDPE is nonpolar. In such system, strong interfacial bond (e.g., hydrogen bond) does not form between LDPE and starch. Furthermore, the presence/absorption of moisture by the starch at the LDPE-starch interface weakens the weak interfacial adhesion. Therefore, the starch granules do not elongate along with the LDPE and thus give rise to easy crack generation and/or propagation eventually resulting in fracture of the polymer sample at lower values of elongation. Figure 1 shows that the drop in elongation at break of the polymer became more prominent as the starch content increased to higher percentage. This could be due to the reason that, at higher starch contents, filler-filler interaction becomes more pronounced than filler-matrix interaction which reduces the effective cross-sectional area of the polymer sample caused by the presence of starch particles. The applied stress is not transferred accordingly from the polymer matrix to the rigid starch particles, and hence the effective stress experienced by the matrix is essentially higher [19].

Unlike the elongation, Young's modulus of the synthesized polymer increased with the increase of starch content (Figure 2). As mentioned, with the increase of starch content the filler-filler interaction becomes more pronounced than filler-matrix interaction, which leads to the agglomeration of starch granules that are inherently more rigid or stiffer than the LDPE matrix [28]. Rigid filler is generally known to increase the modulus of a blend even in the case where true 


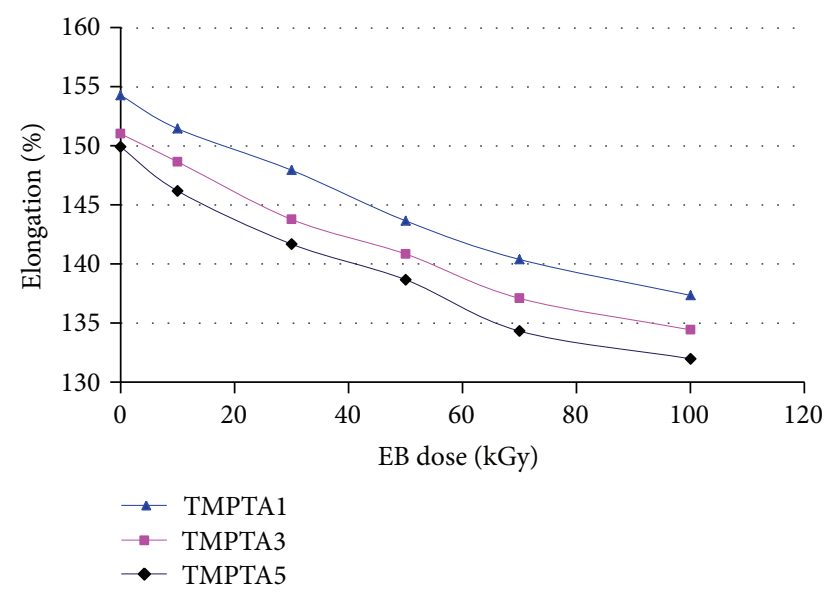

FIgURE 3: Variation of elongation with TMPTA cross-linker and electron beam irradiation.

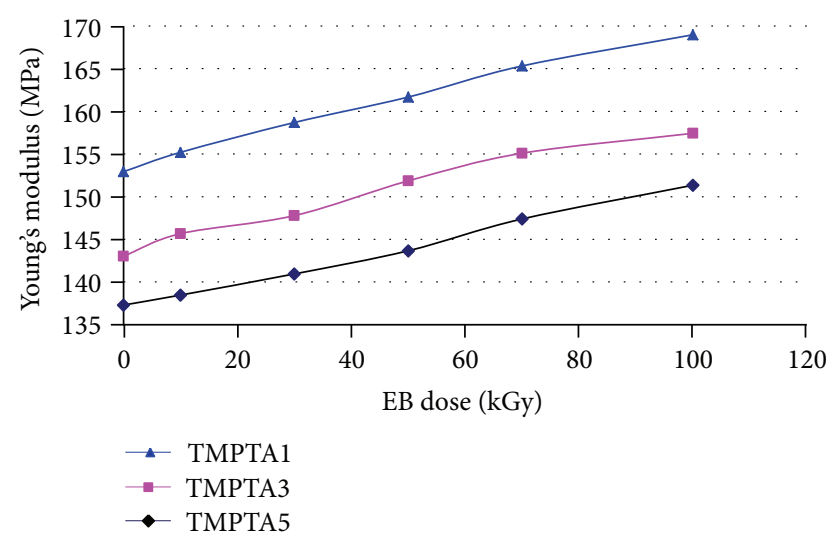

FIGURE 4: Variation of Young's modulus with TMPTA cross-linker and electron beam irradiation.

reinforcement does not occur [24]. Therefore, the increase in modulus of the polymer blend with the increase of starch content is considered to be due to the higher stiffness of the starch granules.

It was observed that the increase of TMPTA crosslinker resulted in decrease of elongation and consequently, increased Young's modulus as shown in Figures 3 and 4. Likewise, the increase of EB irradiation dose also decreased the elongation and thus increased Young's modulus. It is believed that the variation of starch content, cross-linker, and EB irradiation influenced the bond strength of the polymer. As a result, the polymer's mechanical properties were accordingly affected. For example, the elongation became tougher, thus caused higher Young's modulus. However, the detail explanations of these phenomena could be confirmed through intensive bond strength analysis that is out of scope in this preliminary study.

3.2. Thermal Property. The effects of TMPTA cross-linker and EB irradiation on the thermal property (melting temperature) of the polymer were determined via DSC analysis.

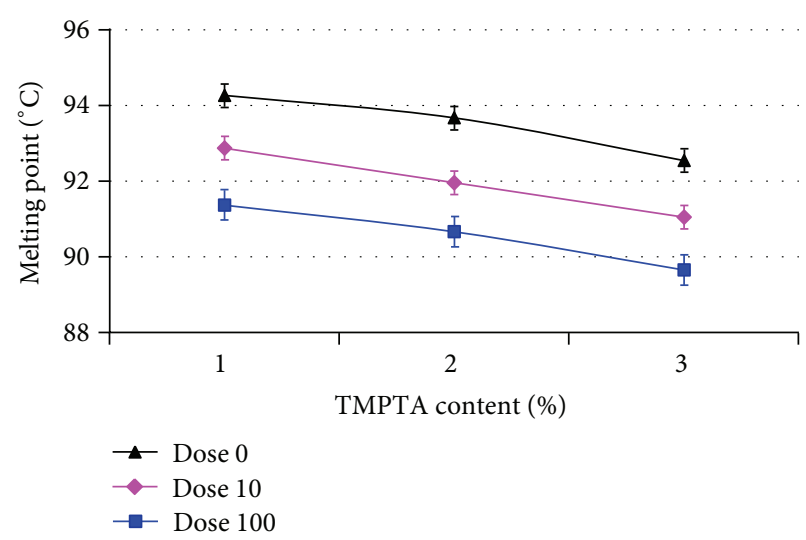

FIGURE 5: Influence of TMPTA cross-linker and electron beam irradiation on the polymer's melting temperature.

TABLE 3: The mass of LPDE polymer samples (before and after degradation) containing various percentages of starch.

\begin{tabular}{lccc}
\hline $\begin{array}{l}\text { Starch } \\
\text { Content (\%) }\end{array}$ & $\begin{array}{c}\text { Mass (before } \\
\text { degradation) (g) }\end{array}$ & $\begin{array}{c}\text { Mass (after } \\
\text { degradation) }(\mathrm{g})\end{array}$ & $\begin{array}{c}\text { Mass loss } \\
(\%)\end{array}$ \\
\hline 0 & 0.464 & 0.464 & 0.00 \\
10 & 0.452 & 0.452 & 0.05 \\
30 & 0.492 & 0.496 & 3.81 \\
50 & 0.53 & 0.528 & 5.37 \\
70 & 0.632 & 0.562 & 11.07 \\
\hline
\end{tabular}

The overall influence of TMPTA cross-linker and EB irradiation on the polymer's melting temperature is presented in Figure 5. The DSC result shows that the increase of EB dosage lowered the polymer's melting points. Similarly, the increase of TMPTA cross-linker also lowered the melting points. The graph illustrates that all the polymer samples modified with TMTA crosslinker and EB irradiation had lower melting points compared to the samples without any modification. As discussed in the previous section, the cross-linker and EB irradiation could have influenced the bond strength of the polymer which, consequently, affected the polymer's melting temperature.

3.2. Thermal Property. Technically, all polymers regardless of their chemical structure or origin degrade under appropriate conditions. However, the term "nondegradable polymer" is meant to indicate the polymer that does not degrade during use or even after very long-term use (e.g., decades to centuries) rather than not to degrade at all [29-31]. The primary rationale of this in vitro degradation study was to understand the degradation behavior and kinetics of the synthesized LPDE polymer and investigate the variation of degradation kinetics due to the variation of starch content in the polymer. The measured masses before and after degradation of the polymer samples are presented in Table 3 from which ultimately the mass loss (i.e., degradation) is calculated.

Pure LDPE and low starch containing (e.g. 10\%) LDPE showed no apparent mass loss. This result is in agreement 


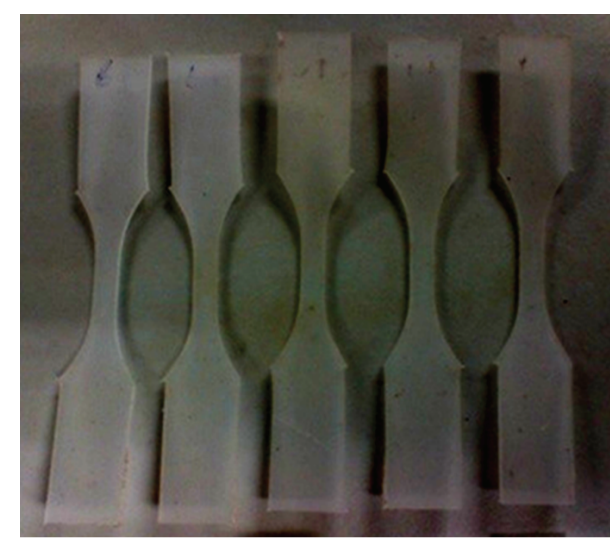

(a)

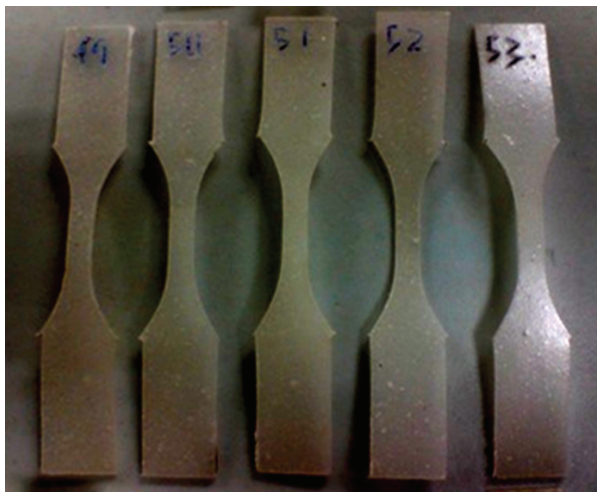

(c)

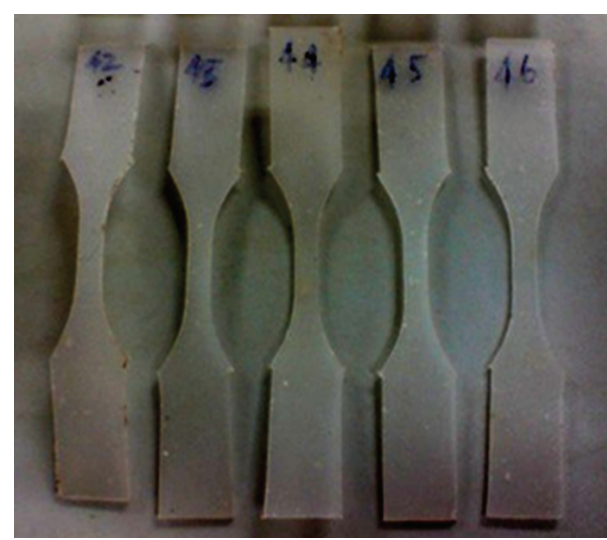

(b)

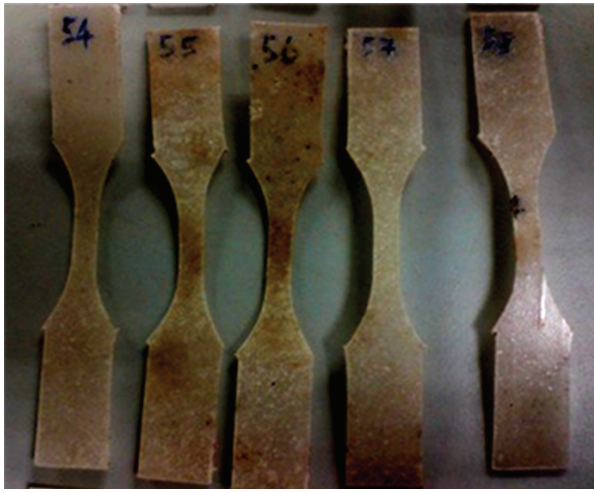

(d)

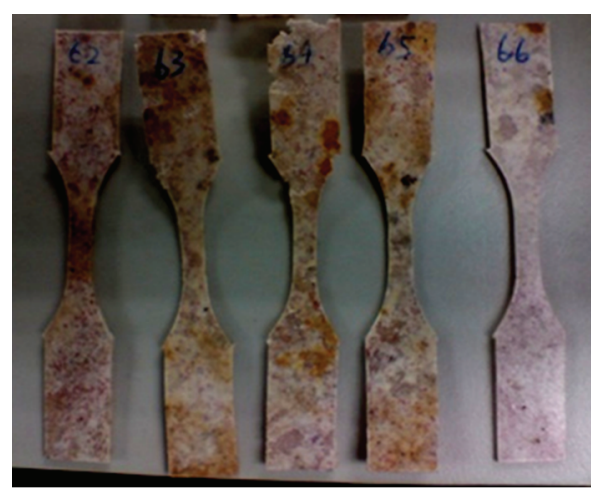

(e)

FIGURE 6: Physical appearances of LDPE polymer samples containing various percentages of starch after a one-month long burial that demonstrate different levels of degradation: (a) Pure LDPE, (b) LDPE 90 and Starch 10, (c) LDPE 70 \& Starch 30, (d) LDPE 50 \& Starch 50, and (e) LDPE 30 \& Starch 70.

with the fact that the pure LDPE is considered to be technically non-degradable, and there should not be any mass loss. Virtually the LDPE polymer sample containing $10 \%$ starch should demonstrate some mass loss (i.e., degradation). However, due to time constraints (e.g., too short burial duration) the degradation might not be observed appreciably. Besides, the presence of starch (only 10\%) might be too low to render the polymer degrade. The LDPE polymer samples containing $30 \%, 50 \%$, and $70 \%$ starch demonstrated mass loss of $3.81 \%, 5.37 \%$, and $11.07 \%$, respectively. These results reveal that higher starch content enhances the degradation kinetics and thus increases mass loss. This could be due to the hydrophilic nature of starch that has been argued in another study [6]. The starch being hydrophilic in nature retains moisture that contributes to the degradation of the polymer. The higher the starch content in the polymer, the higher the moisture content that renders faster degradation. The polymer sample's gross morphology was observed to be changed physically; for example, the surface roughened over the degradation period as seen in Figure 6. The increased starch content resulted in higher surface roughness of the samples due to increased degradation. The weight loss and 
change in physical appearance (i.e., surface roughness) of the sample in the soil could be considered as an evidence of biodegradation of this polymer in the landfills or natural environment. The results indicate that the incorporation of hydrophilic starch into hydrophobic LDPE enhances the hydrophilicity and degradability of the overall polymer. Similar degradation phenomena of the LDPE incorporated with starch were observed in some other studies [20, 25, 27]. Therefore, the degradation characteristic of the starchmixed LDPE polymer could be modulated by manipulating the starch content in the polymer. Indeed, the polymer should be developed with essentially a controlled degradation characteristic while maintaining the required strength of the polymeric object during its designed life time for a particular application.

\section{Conclusions}

The LDPE polymer was successfully synthesized incorporating sago starch and utilizing some modification processes like, TMPTA cross-linker and EB irradiation. The overall process provides the freedom to develop a large variety of polymers with various mechanical, thermal, and degradation properties. The characterization results indicate that the variations of starch content, percentage of TMPTA crosslinker, and EB irradiation dose are very effective means to modulate the polymer's mechanical, thermal, and degradation properties. The polymer's strength (i.e., Young's modulus) increased with the increase of starch content, percentage of TMPTA, and EB irradiation dose, while the ductility of the polymer decreased with the increase of the said parameters. The melting temperature decreased with the increase of TMPTA percentage and EB irradiation dose. The degradation of the polymer was enhanced with the increase of starch content. In conclusion, the starch-incorporated LDPE polymer properties could be modulated by manipulating the starch content and modification processes (e.g., TMPTA crosslinker and $\mathrm{EB}$ irradiation dose) for tailored applications.

\section{Conflict of Interests}

The authors would like to clarify that there is no direct financial relation with the software and corporation mentioned in this paper that might lead to a conflict of interests for any of the authors.

\section{References}

[1] G. Swift, "Requirements for biodegradable water-soluble polymers," Polymer Degradation and Stability, vol. 59, no. 1-3, pp. 19-24, 1998.

[2] P. M. Subramanian, "Plastics recycling and waste management in the US," Resources, Conservation and Recycling, vol. 28, no. 3-4, pp. 253-263, 2000.

[3] V. A. Fomin, "Biodegradable polymers, their present state and future prospects," Progress in Rubber and Plastics Technology, vol. 17, no. 3, pp. 186-204, 2001.

[4] R. Leaversuch, "Biodegradable polyesters: packaging goes green,” Plastics Technology, vol. 48, no. 9, pp. 66-78, 2002.
[5] P. Mukhopadhyay, "Emerging trends in plastic technology," Plastics Engineering, vol. 58, pp. 28-35, 2002.

[6] K. F. Mulder, "Sustainable consumption and production of plastics?" Technological Forecasting and Social Change, vol. 58, no. 1-2, pp. 105-124, 1998.

[7] J. Chen, Y. Maekawa, M. Asano, and M. Yoshida, "Double crosslinked polyetheretherketone-based polymer electrolyte membranes prepared by radiation and thermal crosslinking techniques," Polymer, vol. 48, no. 20, pp. 6002-6009, 2007.

[8] B. Ratner, A. Hoffman, F. Schoen, and J. Lemons, Biomaterials Science: An Introduction to Materials in Medicine, Academic Press, San Diego, Calif, USA, 1996.

[9] M. Cerrada, R. Benavente, M. Fernández-García, E. Pérez, J. Campos, and M. Ribeiro, "Crosslinking in metallocene ethylene-co-5,7-dimethylocta-1,6-diene copolymers initiated by electron-beam irradiation," Polymer, vol. 50, pp. 1095-1102, 2009.

[10] C. Götz, U. A. Handge, M. Piatek, M. El Fray, and V. Altstädt, "Influence of e-beam irradiation on the dynamic creep and fatigue properties of poly(aliphatic/aromatic-ester) copolymers for biomedical applications," Polymer, vol. 50, no. 23, pp. 5499$5507,2009$.

[11] A. Magoń and M. Pyda, "Study of crystalline and amorphous phases of biodegradable poly(lactic acid) by advanced thermal analysis," Polymer, vol. 50, no. 16, pp. 3967-3973, 2009.

[12] S. Chang, H. Ismail, and Q. Ashan, "Effect of maleic anhydride on kenaf dust filled polycaprolactone/thermoplastic sago starch composites," Bioresources, vol. 7, pp. 1594-1616, 2012.

[13] M. Wahab, H. Ismail, and N. Othman, "Compatibilization effects of PE-g-MA on mechanical, thermal and swelling properties of high density polyethylene/natural rubber/thermoplastic tapioca starch blends," Polymer-Plastics Technology and Engineering, vol. 51, pp. 298-303, 2012.

[14] A. Zuraida, Y. Yusliza, H. Anuar, and R. Mohd Khairul Muhaimin, "The effect of water and citric acid on sago starch bio-plastics," International Food Research Journal, vol. 19, no. 2, pp. 715-719, 2012.

[15] A. Zuraida, Y. Yusliza, O. Nurizan, H. Anuar, H. Zahurin, and S. Noorasikin, "Properties of montmorillonite-reinforced thermoplastic sago starch composites," Advanced Materials Research, vol. 445, pp. 469-474, 2012.

[16] D. Wise, Biomaterial and Bioengineering Handbook, Marcel Dekker, New York, NY, USA, 2000.

[17] E. M. Salmoral, M. E. Gonzalez, and M. P. Mariscal, "Biodegradable plastic made from bean products," Industrial Crops and Products, vol. 11, no. 2-3, pp. 217-225, 2000.

[18] O. Martin, E. Schwach, L. Averous, and Y. Couturier, "Properties of biodegradable multilayer films based on plasticized wheat," Starch, vol. 53, pp. 372-380, 2001.

[19] R. Chandra and R. Rustgi, "Biodegradable polymers," Progress in Polymer Science, vol. 23, no. 7, pp. 1273-1335, 1998.

[20] M. Borghei, A. Karbassi, S. Khoramnejadian, A. Oromiehie, and A. H. Javid, "Microbial biodegradable potato starch based low density polyethylene," African Journal of Biotechnology, vol. 9, no. 26, pp. 4075-4080, 2010.

[21] S. M. Goheen and R. P. Wool, "Degradation of polyethylenestarch blends in soil," Journal of Applied Polymer Science, vol. 42, no. 10, pp. 2691-2701, 1991.

[22] J. L. Willett, "Mechanical properties of LDPE/granular starch composites," Journal of Applied Polymer Science, vol. 54, no. 11, pp. 1685-1695, 1994. 
[23] B. G. Kang, S. H. Yoon, S. H. Lee, J. E. Yie, B. S. Yoon, and M. H. O. Suh, "Studies on the physical properties of modified starchfilled HDPE film," Journal of Applied Polymer Science, vol. 60, no. 11, pp. 1977-1984, 1996.

[24] I. Danjaji, R. Nawang, U. Ishiaku, H. Ismail, and Z. Ishak, "Sago starch-filled linear low-density polyethylene (LLDPE) films: their mechanical properties and water absorption," Journal of Applied Polymer Science, vol. 79, pp. 29-37, 2001.

[25] I. D. Danjaji, R. Nawang, U. S. Ishiaku, H. Ismail, and Z. Ishak, "Degradation studies and moisture uptake of sago-starch-filled linear low-density polyethylene composites," Polymer Testing, vol. 21, no. 1, pp. 75-81, 2002.

[26] N. Sharmaa, H. Ismaila, and Z. Ishaka, "Mechanical, water absorption and enzymatic degradation properties of sago starch filled linear low density polyethylene," International Journal of Polymeric Materials, vol. 47, pp. 351-366, 2000.

[27] W. A. W. Abdul Rahman, R. R. Ali, and N. Zakaria, "tudies on biodegradability, morphology and mechanical properties of low density polyehtylene/sago based blends," in Proceedings of the 1st International Conference on Natural Resources Engineering \& Technology, Putrajaya, Malaysia, July 2006.

[28] R. Nawang, I. D. Danjaji, U. S. Ishiaku, H. Ismail, and Z. A. Mohd Ishak, "Mechanical properties of sago starch-filled linear low density polyethylene (LLDPE) composites," Polymer Testing, vol. 20, no. 2, pp. 167-172, 2001.

[29] A. Gopferich, "Mechanisms of polymer degradation and erosion," Biomaterials, vol. 17, pp. 103-114, 1996.

[30] J. Pan, X. Han, W. Niu, and R. E. Cameron, "A model for biodegradation of composite materials made of polyesters and tricalcium phosphates," Biomaterials, vol. 32, no. 9, pp. 22482255, 2011.

[31] R. E. Sallach, W. Cui, F. Balderrama et al., "Long-term biostability of self-assembling protein polymers in the absence of covalent crosslinking," Biomaterials, vol. 31, no. 4, pp. 779-791, 2010 . 

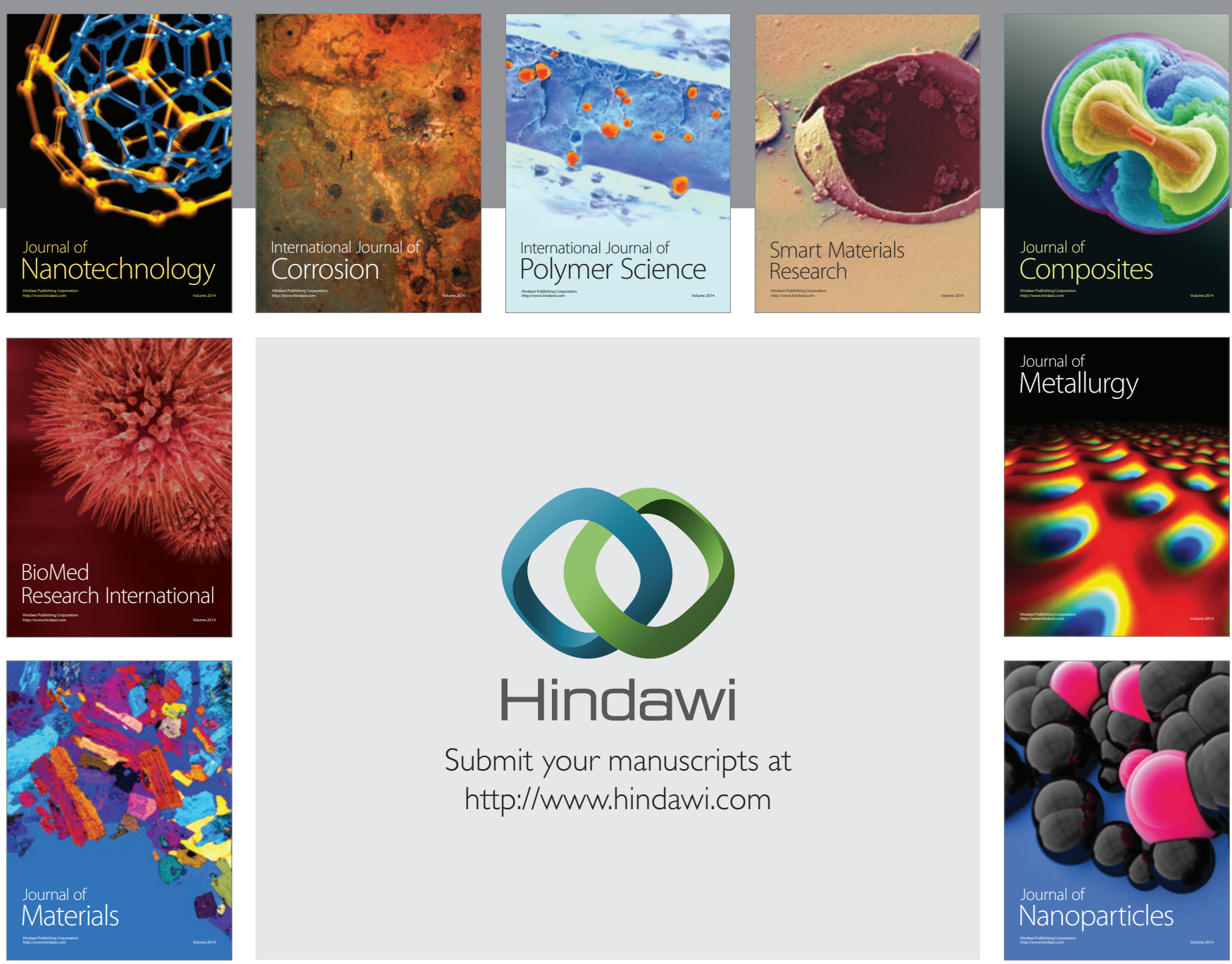

Submit your manuscripts at http://www.hindawi.com
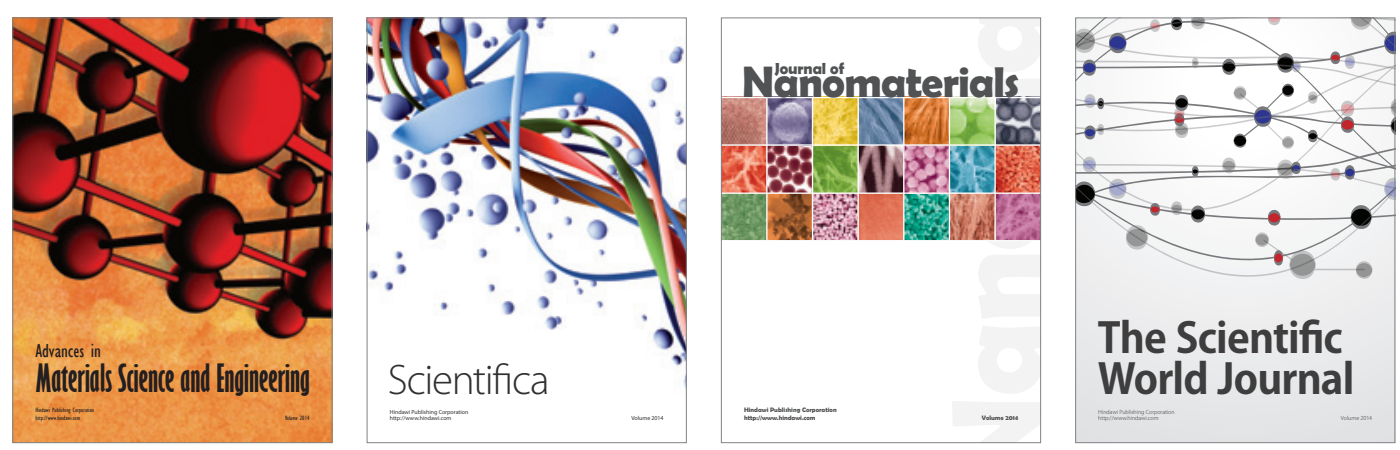

\section{The Scientific World Journal}
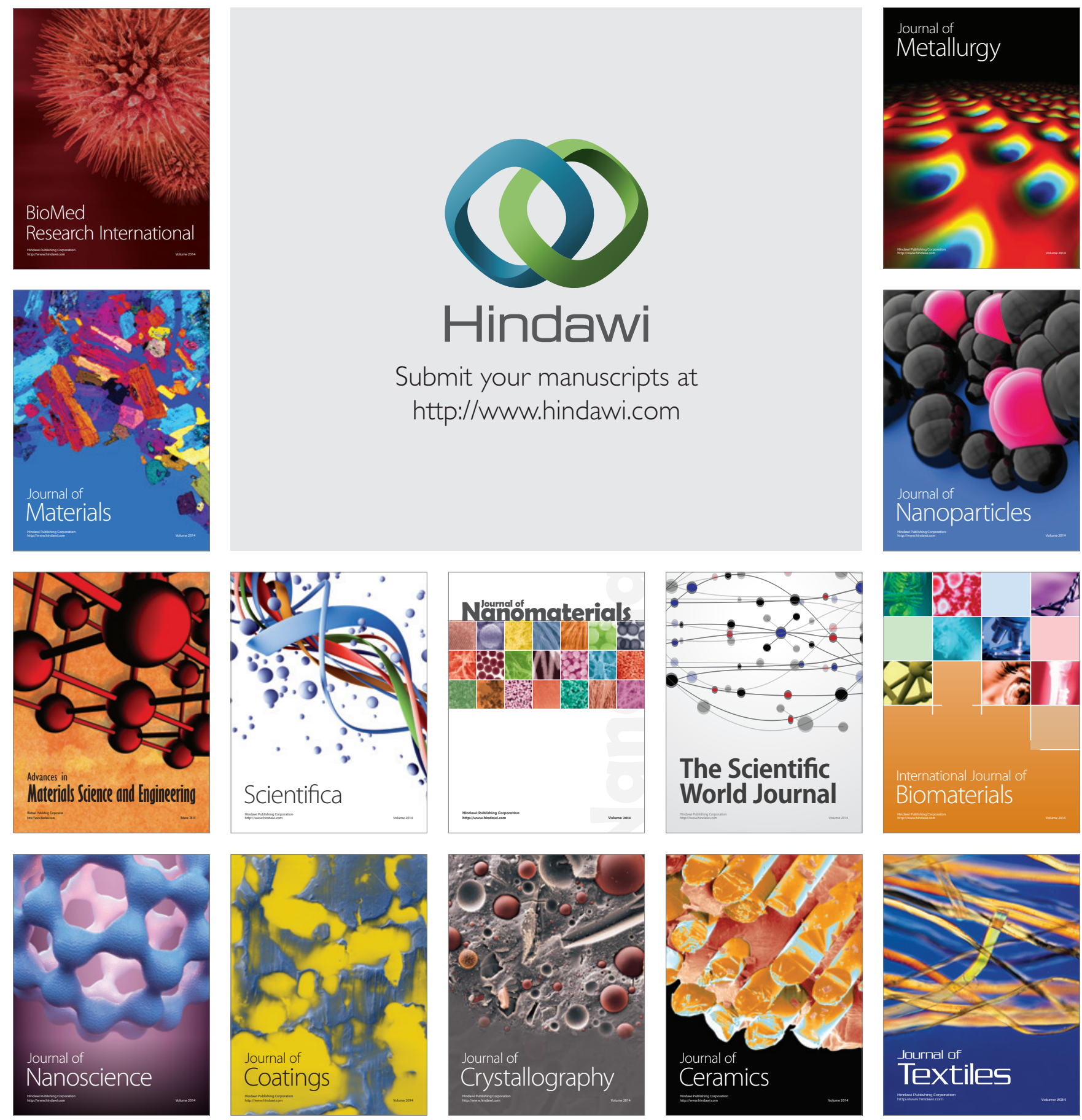